\title{
Differential induction of ATF3 and HO-1 in myeloid cells and keratinocytes via Dimethylfumarate or Cyclosporine A
}

\author{
UNIVERSITÄTS \\ Stefanie Müller, Nikola Smatlik, Marc Burian, Kamran Ghoreschi, \\ Martin Röcken and Amir Yazdi \\ TÜBINGEN
}

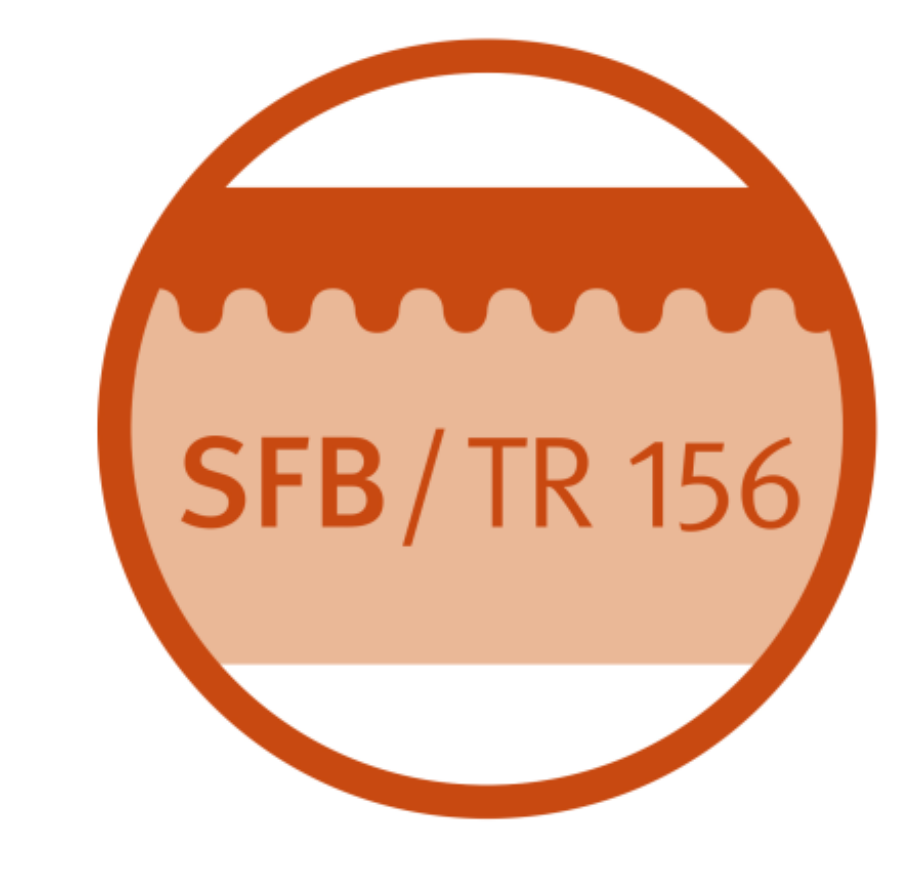

Department of Dermatology, Eberhard Karls University, Liebermeisterstr. 25, D-72076 Tübingen, Germany

\section{Introduction}

Most inflammatory skin diseases such as psoriasis or atopic dermatitis are characterized by skin inflammation resulting in skin hyperplasia. Normally this hyperplasia is well controlled and does not lead to uncontrolled proliferation and to cancer. One key factor which controls and regulates proliferation is the activating transcription factor 3 (ATF3).

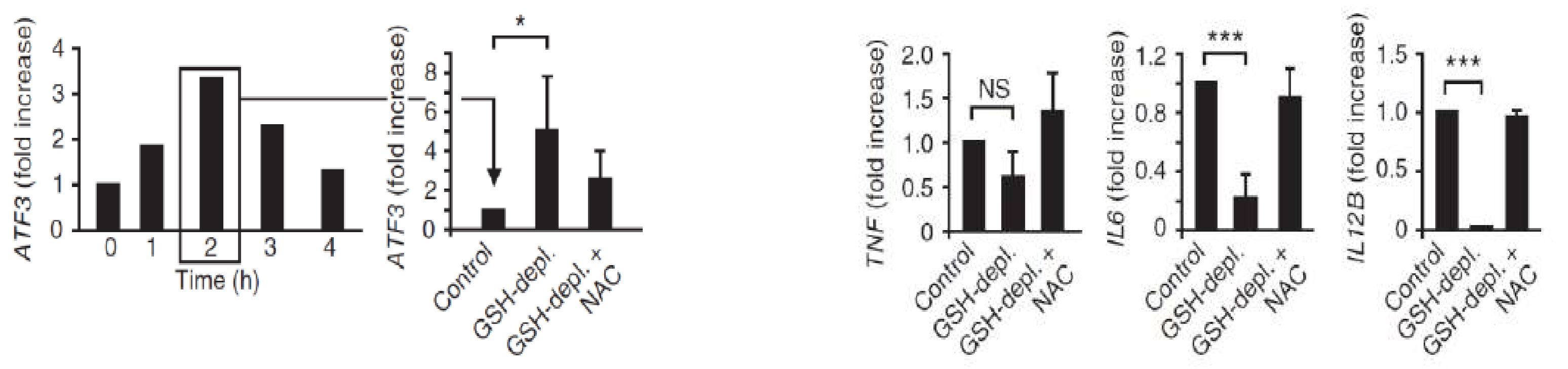

Fig.1: Superinduction of ATF3 by a TLR4-ligand (LPS) and suppression of TNF, IL-6, LL-12B mRNA mediated by increasing ROS via glutathione (GSH) depletion on macrophages (Hötzenecker et al., Nat Med, 2012).

ATF3 is regulated either transcriptionally or via reactive oxygen species (ROS) (Fig.1). This mechanism is therapeutically exploited by the use of fumaric acid in the treatment of psoriasis. Surprisingly, recent data suggest that the IL-6-regulating transcription factor ATF3 has a second role in inflammation-induced skin hyperplasia: On the one hand ATF3 may attenuate inflammation-induced skin hyperplasia by inhibiting chronic inflammation through the repression of IL-6 (Fig.2) and of IL-12p40

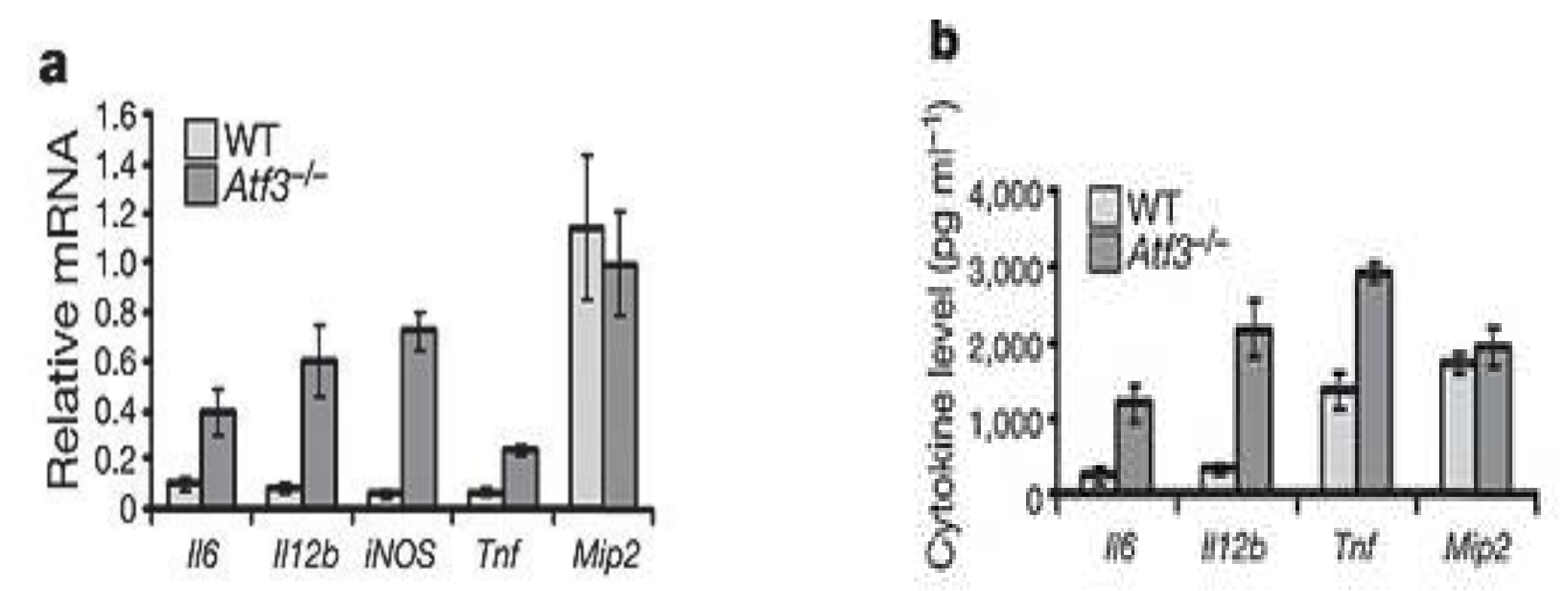

Fig.2: BMDMs from WT and ATF3 ko mice were stimulated with $10 \mathrm{ng} \mathrm{m}^{-1} 1$ LPS for $4 \mathrm{~h}$ and the indicated cytokines were measured by quantitative real-time RT-PCR analysis (Gilchrist et al., Nature, 2006). ATF 3 ko mice show a higher expression of IL-6 on mRNA and protein level.

On the other hand ATF3 may promote keratinocyte proliferation by direct repression of $\mathrm{p} 53$ and interferondominated immune responses that attenuate tissue proliferation. Besides the indirect effect mediated by proinflammatory cytokines, ATF3 might directly influence cell proliferation by binding to the p53 promoter. However, tumor development after chronic inflammatory skin diseases or treatment with fumaric acid is a very rare event. However, cyclosporin A (CSA) which is a potent inducer of ATF3 (Fig.3) is associated with increased rare event. However, cyclosporin A (CSA) which is a potent inducer of ATF3 (Fig.3) is associated with increased
risk of epithelial skin cancer. Here, the ATF3-mediated inhibition of growth-promoting cytokines dominates p53inhibition.
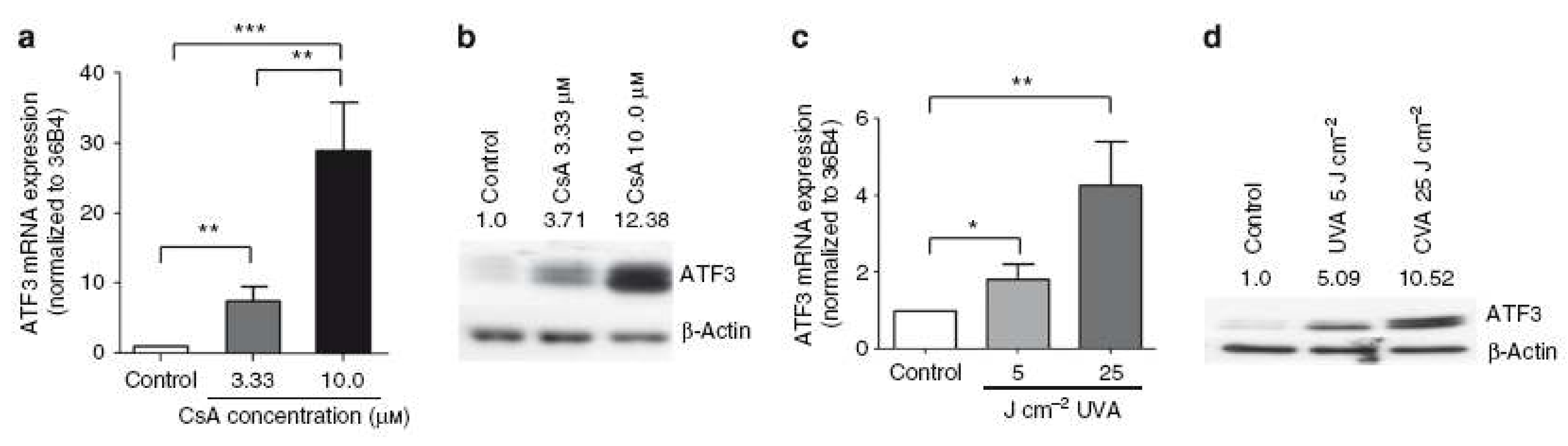

Fig.3: Keratinocytes treated with CSA or exposed to UV radiation. Expression of ATF3 was
(Dzuinyvz, I Invest Derm, 2015). Both treatments show an induction of ATF3 expression.

\section{Results}

To dissect the bi-modal role of ATF3 we pharmacologically induced ATF3 with CSA, a known inducer of ATF3, DMF or Ebselen, and analyzed its influence on cytokine expression and secretion in a cell type specific manner comparing myeloid cells with keratinocytes. DMF is used in the treatment of psoriasis and multiple sclerosis. Ebselen is a glutathione peroxidase mimetic and ROS scavenger, whose anti-inflammatory potential is currently clinically evaluated.

Regulation of the ATF3 and HO-1 induction in human PBMCs and keratinocytes by DMF, Ebselen and Fig. 5 A Cyclosporin A
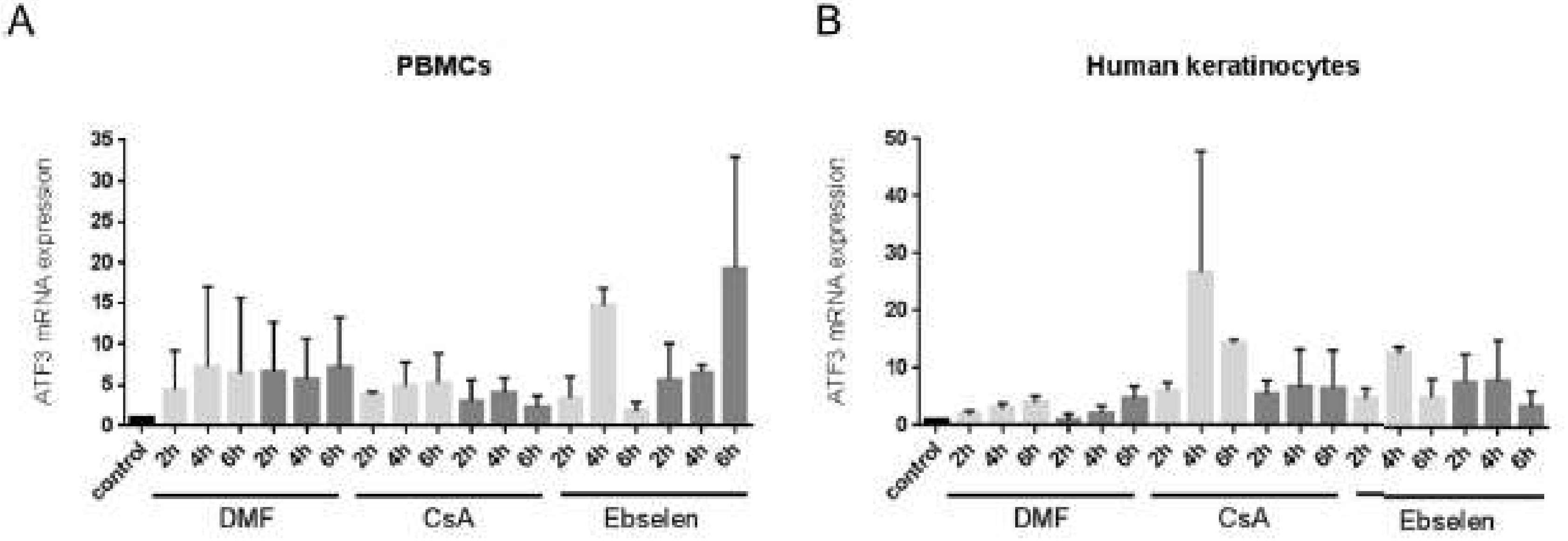

Analyzing the direct ATF3-mRNA-induction primary human keratinocytes and PBMCs were treated with DMF $(70 \mu \mathrm{M}, 35 \mu \mathrm{M}), \mathrm{CsA}(10 \mu \mathrm{M}, 5 \mu \mathrm{M})$ and Ebselen $(20 \mu \mathrm{M}, 10 \mu \mathrm{M})$ for 2 to 6 hours. Interestingly, Ebselen most prominently induced ATF3 in a both time and dose dependent manner in PBMCs, while CsA and DMF only induced a low amount of ATF3(Fig. 5a). Surprisingly, ATF3 induction in keratinocytes differed strongly from ATF3 regulation in PBMCs. CsA most strongly in keratinocytes enhanced ATF3 expression (Fig. 5b). Fig.5 C
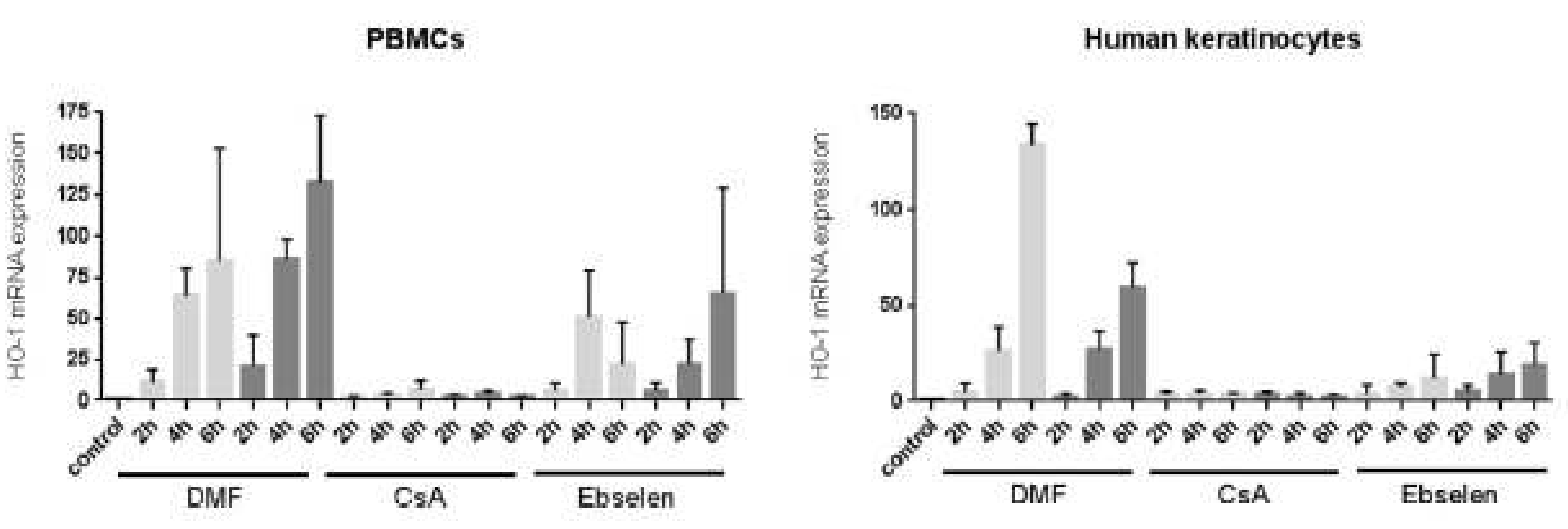

Because of the fact that HO-1 and ATF3 are both induced by cellular stress, we studied the correlation between ATF3 induction and HO-1 increase in myeloid cells and in keratinocytes. In human PBMCs DMF increased both ATF3 (Fig. 5a) and HO-1 mRNA (Fig. 5c) . The ROS-scavenger Ebselen also elevated the transcription of HO-1 and of ATF3. As Ebselen and DMF regulated ATF3 and HO-1 mRNA similarly, the data suggest a link between GSHdepletion and HO-1-induction. In line with this, CSA, the weakest inducer of ATF3 in myeloid cells which does

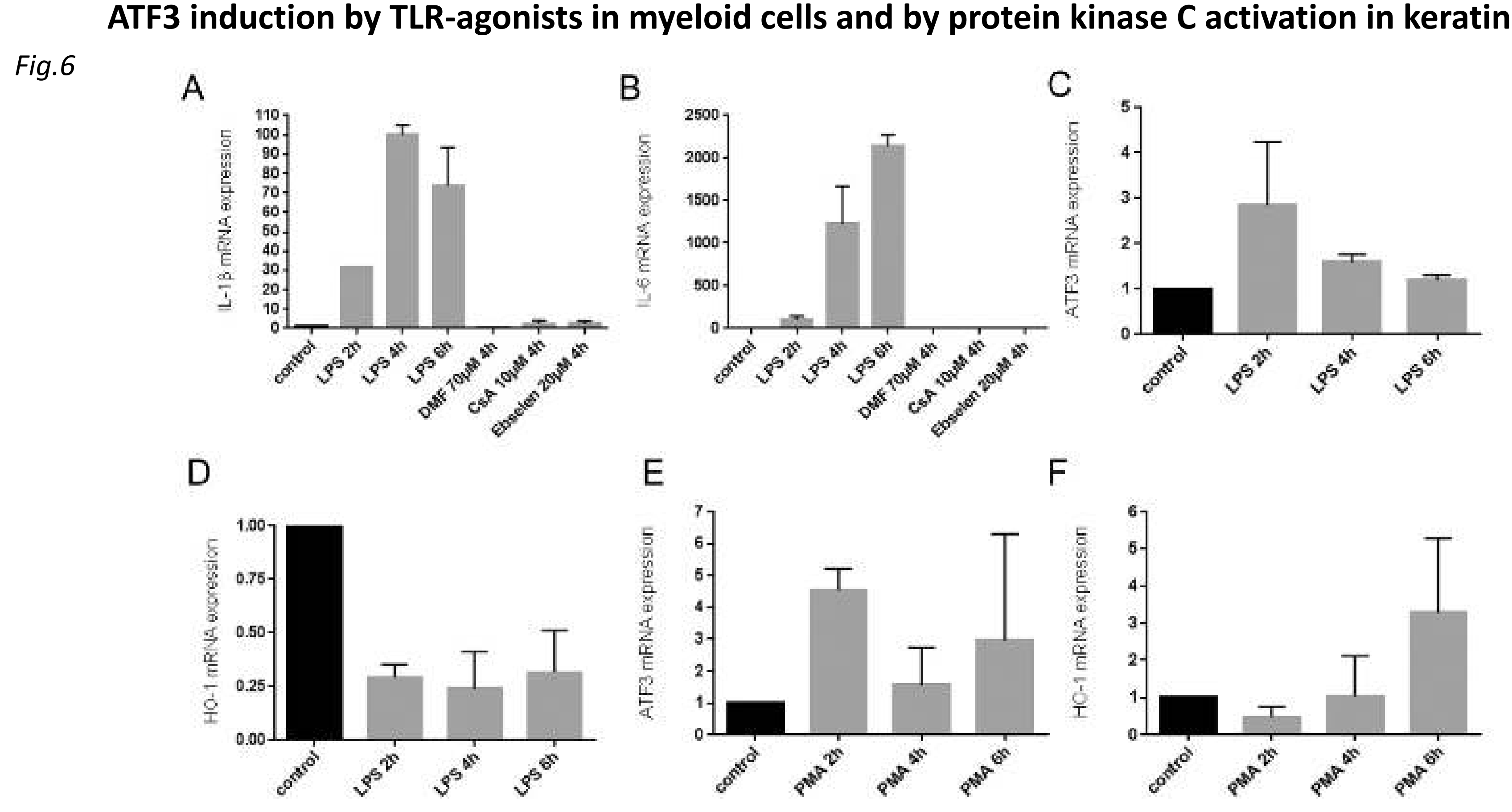

ATF3 was first characterized as a suppressor of TLR4-induced transcription of IL-6 and related cytokines. As ATF3 can also be induced or enhanced in stress responses, we investigated ATF3 expression in response to TLR activation or protein kinase C (PKC) activation by 12-0-Tetradecanoylphorbol-13-acetate (TPA, phorbol 12myristate 13-acetate, PMA). As expected, TLR4 activation by LPS resulted in an increased transcription of the pro-inflammatory cytokines pro-IL-1B (Fig. 6a) and IL-6 (Fig. 6b). Additionally, TLR4 signaling induced a nearly increase in ATF3 expression that peaked after $2 \mathrm{~h}$ (Fig. 6c). TLR-mediated ATF3 transcription did not correlate with HO1-mRNA expression, but rather impaired HO-1 expression in myeloid cells (Fig. 6d). In human primary keratinocytes PMA-treatment leads to an increase in ATF3 which peaked after $2 \mathrm{~h}$ (Fig. 6e) independently of HO1 (Fig. 6f).

Inhibition of LPS-mediated transcription of IL-6, IL-1 $\alpha$ and IL-1 $\beta$ by DMF in myeloid cells
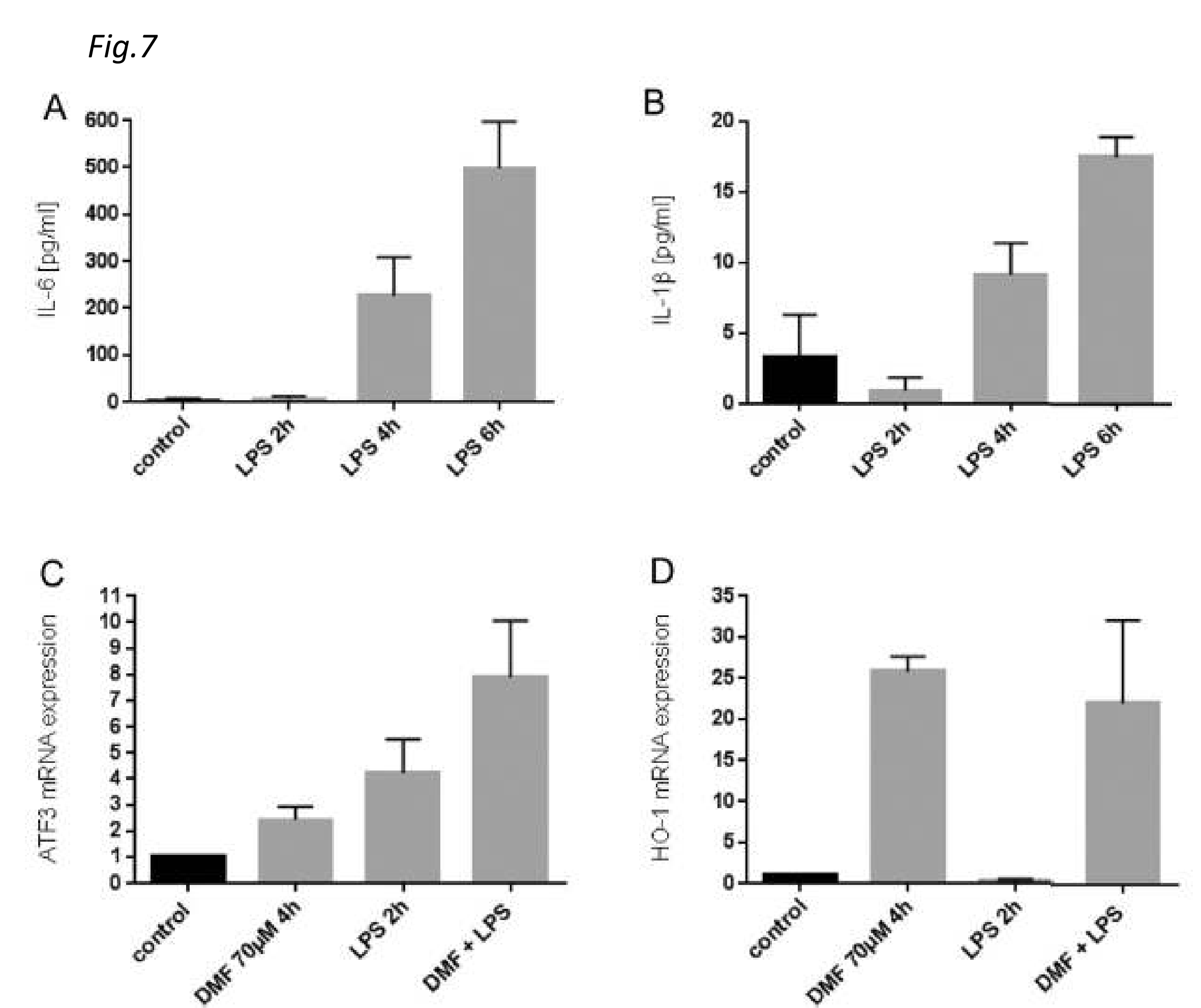

Pro-IL-1 $\alpha$, pro-IL-1 $\beta$ and IL-6 must be transcriptionally induced in myeloid cells by cytokines or TLR-agonists. As ATF3 is known to suppress TLR4-induced IL-6 transcription we next investigated the effect of ATF3-induction on TLR-induced transcription of pro-inflammatory mediators. Without additional TLR-activation, ATF3 induction by DMF alone neither amplified nor diminished pro-IL-1 or IL-6 transcription, while LPS treatment induced the transcription of these cytokines (Fig. 6a, Fig. 6b) in PBMCs. In line with published data, IL-6, which was secreted directly after its MRNA induction by TLR4 activation (Fig. 7a), TLR activation alone induced the secretion of only very low amounts of IL-1B (Fig. 7b).

Fig.8

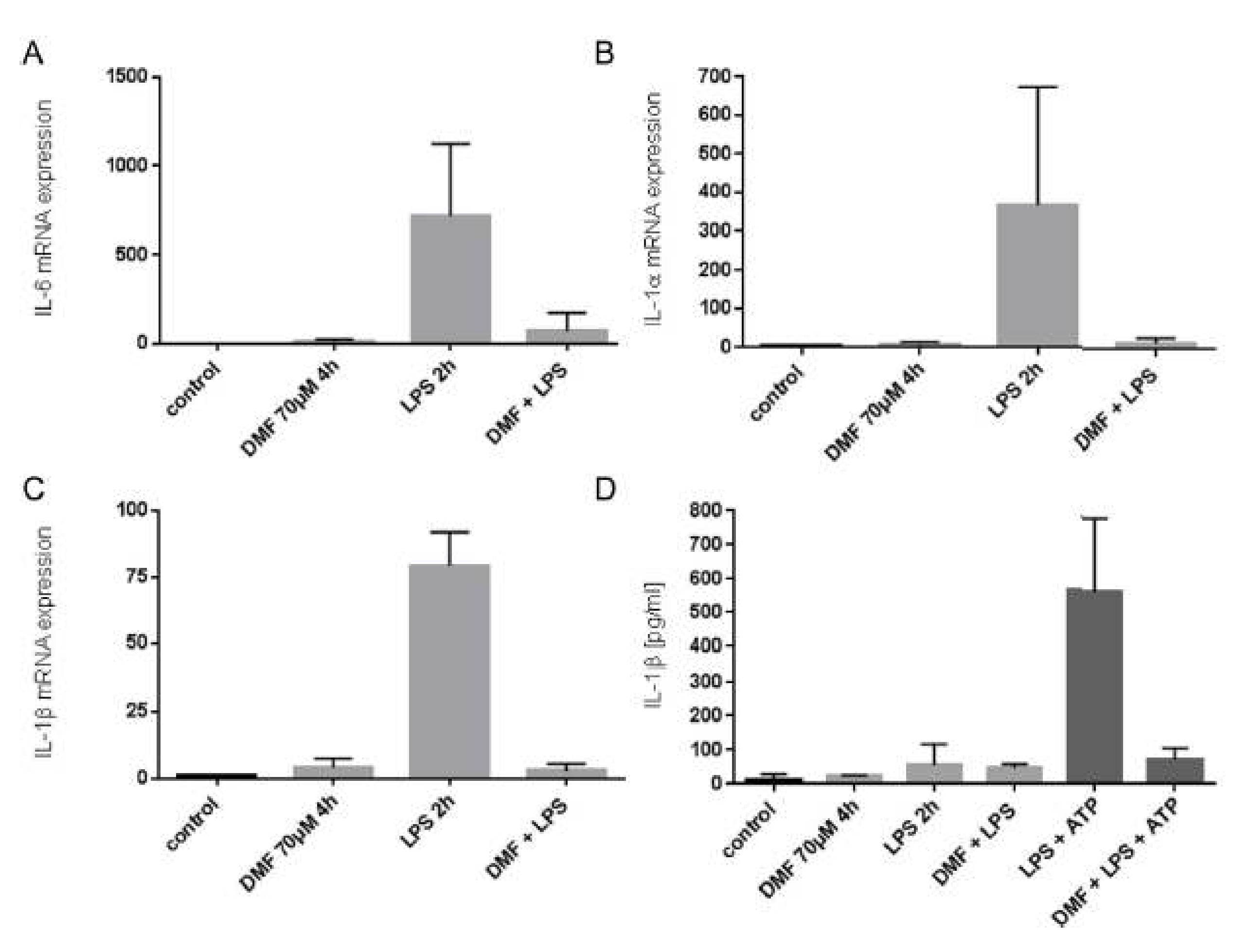

In most cell types, the secretion of IL-1 $\alpha$ and IL- $1 \beta$ is regulated at two steps: First the full-length cytokines are transcriptionally induced. The subsequent IL-1B secretion requires a second signal. This second signal is usually a Nod-like receptor ligand. However, in human myeloid cells stimulation by a TLR ligand is sufficient to induce a very low IL-1 secretion. If cells are pretreated prior to LPS-treatment with DMF, ATF3 was superinduced (Fig. 7c) while HO-1 (Fig. 7d) remained unaltered. In consequence, DMF was capable of drastically reducing LPS-induced production of IL-6 (Fig. 8a), IL-1 $\alpha$ (Fig. 8b) or IL-1 $\beta$ (Fig. 8c), respectively. To investigate the impact of NLRP3 activation, PBMCs were additionally treated with the NLRP3 agonist ATP. Due to less pro-IL-1 $\beta$ transcribed, DMF also dramatically reduced IL-1 $\beta$ secretion by myeloid cells (Fig. 8d).

\section{Conclusion}

Taken together, our results shed light into the different carcinogenic potential of CSA and DMF, which both target ATF3. While keratinocytes are more susceptible to CsA-induced ATF3, the anti-inflammatory potential of DMF dominates in myeloid cells. To investigate the complex interplay between tumor-promoting inflammation in the tumor-associated infiltrate and $\mathrm{p53-controlled} \mathrm{proliferation} \mathrm{in} \mathrm{keratinocytes,} \mathrm{murine} \mathrm{models} \mathrm{are} \mathrm{needed.}$ Especially ATF3 and IL-6 knock-out mice will help to decipher the interplay between anti-inflammatory effects of ATF3 and the pro-tumorigenic suppression of p53-dependent senescence in developing cancer. 